\title{
Papers
}

\section{Eating disorders in adolescent females with and without type 1 diabetes: cross sectional study}

\author{
Jennifer M Jones, Margaret L Lawson, Denis Daneman, Marion P Olmsted, Gary Rodin
}

\begin{abstract}
Objective To determine the prevalence of eating disorders in adolescent females with type 1 diabetes mellitus compared with that in their non-diabetic peers. Design Cross sectional case-control led study. Setting Diabetes clinics and schools in three Canadian cities.

Subjects 356 females aged 12-19 with type 1 diabetes and 1098 age matched non-diabetic controls. Main outcome measure Eating disorders meeting Diagnostic and Statistical Manual of Mental Disorders (DSM-IV) criteria.

Results Eating disorders that met DSM-IV criteria were more prevalent in diabetic subjects $(36,10 \%)$ than in non-diabetic controls $(49,4 \%$ ) (odds ratio $2.4,95 \%$ confidence interval 1.5 to $3.7 ; \mathrm{P}<0.001)$. Subthreshold eating disorders were also more common in those with diabetes $(49,14 \%)$ than in controls $(84,8 \%)$ (odds ratio $1.9,95 \%$ confidence interval 1.3 to $2.8 ; \mathrm{P}<0.001)$. Mean haemoglobin $\mathrm{A}_{1 \mathrm{c}}$ concentration was higher in diabetic subjects with an eating disorder $(9.4 \%(1.8))$ than in those without $(8.6 \%(1.6)), \mathrm{P}=0.04)$.

Conclusions DSM-IV and subthreshold eating disorders are almost twice as common in adolescent females with type 1 diabetes as in their non-diabetic peers. In diabetic subjects, eating disorders are associated with insulin omission for weight loss and impaired metabolic control.
\end{abstract}

\section{Introduction}

Eating disorders are common in late adolescent and young adult women in westernised countries. ${ }^{1-3}$ These conditions are of particular concern in young women with type 1 diabetes mellitus because of their association with impaired metabolic control and an earlier than expected onset of diabetes related complications. ${ }^{4-6}$ Aspects of type 1 diabetes and its treatment that have been postulated to increase the risk of eating disorders in young women include the cycle of weight loss at disease onset and subsequent weight gain with the initiation of insulin treatment, the trend towards higher body mass index, ${ }^{7}$ dietary restraint necessitated by diabetes management, and the availability of deliberate insulin underdosing or omission as a weight loss strategy. ${ }^{8}$ However, whether eating disorders occur more frequently in females with diabetes than their non-diabetic peers is controversial.
Five controlled, interview based prevalence studies of eating disorders in young women with type 1 diabetes have been reported in which the authors concluded that eating disorders were not more common than in similar non-diabetic populations. ${ }^{9-13}$ However, these negative findings were based on small sample sizes of young women in the age group at highest risk for eating disorders and consequently had low statistical power to detect significant differences in prevalence. We compared the prevalence of eating disorders in a large sample of adolescent girls with type 1 diabetes to that in a large age matched, non-diabetic female population to determine whether eating disorders are more common among those with diabetes.

\section{Participants and methods}

Females aged 12 to 19 years who had had type 1 diabetes for at least one year were identified from diabetes clinic lists at the Hospital for Sick Children in Toronto, the Children's Hospital of Eastern Ontario in Ottawa, and the Children's Hospital at Hamilton Health Sciences Corporation in Hamilton, Ontario. The subjects were approached by post and telephone call or during clinic appointments. All three diabetes clinics are the main primary treatment centres in their areas for children and adolescents with type 1 diabetes, providing care for about $70 \%$ of potential patients within their catchment areas.

A comparison group of 2494 female students without diabetes was identified at junior high and high schools in Toronto, Ottawa, and Hamilton. The study was described in the same way to both the students and the diabetic patients. The subjects were approached individually whereas the controls were approached as a group during a class. We obtained research ethics approval for each site and informed written consent from each participant and her parent, when required.

\section{Study protocol}

All participants completed a self report screening package that included the eating disorder inventory, ${ }^{14}$ the eating attitudes test (EAT-26), ${ }^{15}$ and the diagnostic survey for eating disorders (modified) ${ }^{16}$ Body mass index (weight $(\mathrm{kg}) /\left(\right.$ height $\left.(\mathrm{m})^{2}\right)$ ) was calculated based on self reported height and weight. Participants who were considered to be at risk of an eating disorder because they scored above predetermined cut off levels (box) were asked to complete the eating disorder

\author{
Department of \\ Psychiatry, \\ University Health \\ Network, Toronto, \\ Ontario, Canada \\ M5G 2CA \\ Jennifer M Jones \\ research fellow \\ Division of \\ Endocrinology, \\ Children's Hospital \\ of Eastern Ontario, \\ Ottawa, Ontario, \\ Canada K1H 8LI \\ Margaret L Lawson \\ head \\ Division of \\ Endocrinology, \\ Hospital for Sick \\ Children, Toronto, \\ Ontario, Canada \\ M5G 2CA \\ Denis Daneman \\ chief \\ Ambulatory Care \\ for Eating \\ Disorders, \\ University Health \\ Network \\ Marion P Olmsted \\ director \\ Department of \\ Psychiatry, \\ University Health \\ Network \\ Gary Rodin \\ psychiatrist in chief \\ Correspondence to: \\ G Rodin \\ gary.rodin@uhn. \\ on.ca
}

BMJ 2000;320:1563-6

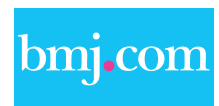

The diagnostic criteria used for eating disorders are given on the BMJ's website 


\section{Screening cut off criteria for interviews}

1. Score of $\geqslant 15$ on drive for thinness subscale of the eating disorder inventory (corresponds to 88th percentile for girls aged 11 to 18 years and 47th percentile for patients with clinically diagnosed eating disorder ${ }^{14}$ ) 2. Score of $\geqslant 5$ on bulimia subscale of the eating disorder inventory (86th percentile for 11 to 18 year old girls and 21 st percentile for patients with eating disorder ${ }^{14}$ )

3. Score of $\geqslant 20$ on body dissatisfaction subscale of the eating disorder inventory (87th percentile for 11 to 18 year old girls and 60 th percentile for eating disorder patients ${ }^{14}$ )

4. Total score of $\geqslant 20$ on the eating attitudes test (recommended cut off score for screening purposes ${ }^{15}$ )

5. Current or past history of binge eating, self induced vomiting, use of laxatives or diuretics, insulin omission for weight loss, or current dietary restriction as assessed by the diagnostic survey for eating disorders

6. History of eating disorder diagnosis or treatment reported in diagnostic survey for eating disorders

7 . $\leqslant 5$ th percentile of body mass index for age matched females ${ }^{19}$

\section{Classification of eating disorders}

The classification of eating disorders was based on data from the eating disorder examination interview. Subjects were classified as having a full syndrome eating disorder based on DSM-IV criteria (anorexia nervosa, bulimia nervosa, or "eating disorder not otherwise specified") $)^{23}$; a subthreshold eating disorder; or no eating disorder. These three categories were mutually exclusive (see BMJ's website for details of diagnostic criteria).

Based on DSM-IV criteria a minimum of four clinical symptoms over the past three months were necessary for the diagnosis of anorexia or bulimia nervosa. DSM-IV provides broad suggestions for diagnosis of eating disorders not otherwise specified. We operationalised the diagnosis of these disorders more precisely based on DSM-IV criteria and criteria used in previous controlled prevalence studies. ${ }^{11}{ }^{11}$ Subthreshold eating disorders were considered to be milder eating disturbances with a lower frequency or severity of symptoms over the past three months than those specified in DSM-IV.

(version 11.5d-modified), which is a ser structured diagnostic interview. ${ }^{17}$ An additional 15\% of the subjects who did not meet the screening cut off were randomly selected for the interview to ensure interviewer blindness. Interviewers could not be blind to diabetes status because the questionnaire contained questions relating to diabetes, which we included for the diabetes sample (for example, on insulin omission for weight loss). Blood was obtained by finger prick from each of the diabetic subjects to measure haemoglobin $\mathrm{A}_{1 \mathrm{c}}$ concentrations. All samples were analysed at the Hospital for Sick Children with the BioRad variant high pressure liquid chromatography assay (normal range $4-6 \%)^{18}$

\section{Screening measures}

The eating disorders inventory and eating attitudes test are self administered questionnaires shown to be reliable and valid screening measures for eating disorders in both diabetic and non-diabetic populations. ${ }^{14} 152021$ The eating disorders inventory provides quantitative assessments of specific eating attitudes and behaviours, and the eating attitudes test provides a total score for disturbed eating attitudes and behaviour. Scores on both questionnaires were corrected to discount items that may be scored positively because of diabetes and its treatment (such as "Aware of the calorie content of foods that I eat"). The diagnostic survey for eating disorders is a self administered questionnaire that allows the frequency of disturbed behaviour to be quantified. ${ }^{16}$ We modified this measure to include diabetes related items such as intentional insulin omission for weight loss. ${ }^{42}$ The eating disorders examination is a semistructured diagnostic interview that quantifies the symptoms, behaviour, and psychopathology of eating disorders and allows eating disorders to be diagnosed according to the Diagnostic and Statistical Manual of Mental Disorders (DSM-IV). ${ }^{23}$ This interview has good reliability and validity and is currently considered the gold standard for the standardised assessment of eating disorders. ${ }^{1724-26}$ We used a modified version to evaluate diabetes specific behaviour related to eating disorders and to determine if eating behaviour was motivated by diabetes management or weight and shape reasons.

\section{Statistical analysis}

We used $\chi^{2}$ analyses with Yates's correction for continuity to test the prevalence of clinical and subthreshold eating disorders between the three cities and in the combined samples. Fisher's exact test was used to test the proportions for bulimia. We compared normally distributed continuous variables using Student's $t$ tests and analysis of variance. Scheffe's method for post-hoc comparisons was used to deal with inflated type I error rates when significant effects were found by analysis of variance. Non-normal data were transformed logarithmically and independent $t$ tests were used for comparisons between two groups. Odds ratios and corresponding confidence intervals were calculated to measure the main effect of diabetes on eating disorders. $\mathrm{P}$ values $<0.05$ were considered significant. All $\mathrm{P}$ values are two tailed.

\section{Results}

\section{Participants and non-participants}

In total, 361 of 430 eligible females with type 1 diabetes (84\%) agreed to participate in the study. Of the 2494 eligible school girls without diabetes, 1840 (74\%) returned signed consent forms and agreed to take part in the study, and an additional $151(8 \%)$ subjects were absent or could not participate because of school tests on the day of the survey. Screening data were collected on 1689 subjects without diabetes. Based on projected numbers of diabetic subjects at each site, we randomly selected a subsample of 1114 age and site matched control subjects (roughly 3:1 ratio).

There were no significant differences between the three clinics or between the schools across the three cities. Therefore, all subsequent analyses were based on two groups - that is, combined diabetes and combined control samples. The mean body mass index was higher in the diabetes group (22.7 (SD 3.8) versus 20.6 (3.3), $\mathrm{P}<0.001$ ), and the proportion of subjects from higher socioeconomic groups (IV, V, and VI) was slightly higher among control subjects $(53 \%$ versus $61 \%, \mathrm{P}=0.02)($ table 1$)$ 
Table 1 Demographic characteristics of study participants. Values are mean (SD) unless stated otherwise

\begin{tabular}{lcc} 
Characteristic & $\begin{array}{c}\text { Diabetic subjects } \\
(\mathbf{n}=\mathbf{3 6 1 )}\end{array}$ & $\begin{array}{c}\text { Controls } \\
\mathbf{( n = 1 1 1 4 )}\end{array}$ \\
\hline Age (years) & $14.9(2.0)$ & $14.8(1.9)$ \\
\hline Body mass index & $22.7(3.8)$ & $20.6(3.3)$ \\
\hline No (\%) with socioeconomic statust: & & \\
\hline I $(\geqslant C n \$ 15000)$ & $5(1)$ & $7(1)$ \\
\hline II $(\geqslant C n \$ 25000)$ & $47(13)$ & $81(8)$ \\
\hline III $(\geqslant C n \$ 35000)$ & $114(33)$ & $290(30)$ \\
\hline IV $(\geqslant C n \$ 50000)$ & $148(42)$ & $416(43)$ \\
\hline V $(\geqslant C n \$ 75000)$ & $34(10)$ & $140(14)$ \\
\hline VI $(\geqslant C n \$ 100000)$ & $4(1)$ & $36(4)$ \\
\hline Age at onset of diabetes (years) & $8.1(3.6)$ & - \\
\hline Duration of diabetes (years) & $6.7(3.6)$ & - \\
\hline Haemoglobin $\mathrm{A}_{1 \mathrm{c}}(\%)$ & $8.8(1.7)$ & - \\
\hline
\end{tabular}

${ }^{\star} P<0.001$ for the comparison between diabetes and control subjects. †Socioeconomic status was based on Statistics Canada income tax and postal code data for the 970 control subjects and 352 diabetic subjects who returned demographic forms; $\mathrm{P}=0.01$ for $\chi^{2}$ analysis between diabetic and control subjects. $£ 1=\mathrm{Cn} \$ 2.24$

\section{Self reported data}

Based on the diagnostic survey for eating disorders, diabetic subjects reported more binge eating and less dieting for the purpose of losing weight than controls (table 2). There were no significant differences between the two groups in frequency of self induced vomiting or misuse of laxatives, or on the eating disorder inventory subscales or eating attitudes test total score.

A total of $48 \%$ of the control subjects $(n=536)$ and $52 \%$ of the diabetic subjects $(n=187)$ scored above the predetermined screening cut off and were asked to participate in the eating disorders examination diagnostic interview. Three per cent of both the controls $(n=16)$ and the diabetic subjects $(n=5)$ who met cut off criteria did not participate in the interview and were excluded from the prevalence analysis. In order to keep the interviewer blinded to screening status, an additional 98 control subjects (17\%) and 38 diabetic subjects $(19 \%)$ who did not meet cut off were randomly selected to participate in the interview. In total, $220(61 \%)$ subjects with diabetes and $616(55 \%)$ controls completed the eating disorder examination interview.

\section{Frequency of DSM-IV and subthreshold eating disorders}

Table 3 shows the frequency of eating disorders based on DSM-IV criteria. Subjects with diabetes were 2.4 times more likely than controls to have an eating disorder $(95 \%$ confidence interval 1.5 to 3.7$)$. In total, 36 (10\%) of the 356 diabetic subjects who completed the study met DSM-IV criteria for eating disorders compared with 49 (4\%) of the 1098 non-diabetic controls $(\mathrm{P}<0.001)$. Eating disorder not otherwise specified was the most frequent diagnosis, and there were no cases of anorexia nervosa in either group. Diabetic subjects were also 1.9 times (1.3 to 2.8) more likely to have a subthreshold eating disorder than control subjects $(49(14 \%)$ versus $84(8 \%) ; \mathrm{P}<0.001)$. There was no significant difference in socioeconomic status between eating disorder groups.

Insulin manipulation and haemoglobin $\mathbf{A}_{1 \mathrm{c}}$

In subjects with diabetes, deliberate insulin omission was the most common weight loss behaviour after diet- ing. At screening, $41(11 \%)$ reported that they were currently taking less than their prescribed dose of insulin to lose weight. Fifteen (42\%) of the 36 diabetic subjects with an eating disorder reported insulin misuse at the time of screening, which was significantly higher than the proportion in the subthreshold $(9,18 \%)$ and non-disordered groups $(16,6 \% ; \mathrm{P}<0.001)$. Diabetic subjects with eating disorders had significantly higher mean haemoglobin $\mathrm{A}_{1 \mathrm{c}}$ concentrations $(9.4 \%$ (SD 1.8)) compared with those without an eating disorder $(8.6 \%$ (1.6), $\mathrm{P}=0.04$ ). The mean haemoglobin $\mathrm{A}_{1 \mathrm{c}}$ concentration for subjects with a subthreshold disorder $(9.1 \%$ (1.8)) did not differ significantly from that in the DSM-IV or non-disordered groups.

\section{Discussion}

This multisite case-controlled study has shown that eating disorders that meet DSM-IV diagnostic criteria and their subthreshold variants are about twice as common in adolescent females with type 1 diabetes as in their non-diabetic peers. Among diabetic subjects, the mean haemoglobin $\mathrm{A}_{1 \mathrm{c}}$ concentration was higher in those with an eating disorder than in those without an eating disorder. Such an association between eating disorders and impaired metabolic control may contribute to an increased risk of microvascular complications in young women with diabetes and eating disorders. ${ }^{47}$

Insulin omission, which is recognised as a purging behaviour in the DSM-IV criteria, was the most common weight loss method after dieting among the diabetic subjects. The availability of this method of weight control, together with the dietary restrictions imposed by the diabetes regimen, may explain why the diabetes group reported less dieting to lose weight than the control group, even though they reported more binge eating.

Our study has some limitations. Although the participation rates of $84 \%$ in the diabetes group and $74 \%$

Table 2 Self reported behaviours of diabetic and control groups based on diagnostic survey for eating disorders (modified)

\begin{tabular}{|c|c|c|c|c|}
\hline \multirow[b]{2}{*}{ Variable } & \multicolumn{2}{|c|}{ No $(\%)$ of } & \multirow[b]{2}{*}{$\begin{array}{c}\text { Odds ratio } \\
(95 \% \mathrm{Cl})\end{array}$} & \multirow[b]{2}{*}{ P value ${ }^{*}$} \\
\hline & $\begin{array}{l}\text { Diabetic } \\
\text { subjects } \\
(n=361)\end{array}$ & $\begin{array}{c}\text { Controls } \\
(n=1114)\end{array}$ & & \\
\hline Binge eating & $108(30)$ & $251(23)$ & 1.5 (1.1 to 1.9$)$ & 0.004 \\
\hline Dieting for weight loss & $43(12)$ & $254(23)$ & 0.5 (0.3 to 0.6$)$ & 0.001 \\
\hline Self induced vomiting & $25(7)$ & $95(9)$ & 0.8 (0.5 to 1.3$)$ & 0.333 \\
\hline Laxative use & $9(2)$ & $14(1)$ & 2.0 (0.9 to 4.7$)$ & 0.100 \\
\hline Omission or underdosing of insulin for weight loss & $41(11)$ & - & - & - \\
\hline
\end{tabular}

*For comparison between diabetic subjects and controls by $\chi^{2}$ analysis.

Table 3 Frequency of DSM-IV and subthreshold eating disorders among diabetic subjects and matched controls

\begin{tabular}{|c|c|c|c|c|}
\hline \multirow[b]{2}{*}{ Eating disorder } & \multicolumn{2}{|c|}{ No $(\%)$ of } & \multirow[b]{2}{*}{$\begin{array}{l}\text { Odds ratio } \\
\text { (95\% CI) }\end{array}$} & \multirow[b]{2}{*}{ P value* } \\
\hline & $\begin{array}{l}\text { Diabetic } \\
\text { subjects } \\
(n=356)\end{array}$ & $\begin{array}{l}\text { Controls } \\
(n=1098)\end{array}$ & & \\
\hline DSM-IV disorder & $36(10)$ & $49(4)$ & 2.4 (1.5 to 3.7$)$ & $<0.001$ \\
\hline Anorexia nervosa & 0 & 0 & - & - \\
\hline Bulimia nervosa & $5(1)$ & $5(0.5)$ & 3.1 (0.9 to 10.8$)$ & 0.07 \\
\hline Not otherwise specified & $31(9)$ & $44(4)$ & 2.3 (1.4 to 3.7$)$ & $<0.001$ \\
\hline Subthreshold disorders & $49(14)$ & $84(8)$ & 1.9 (1.3 to 2.8$)$ & $<0.001$ \\
\hline
\end{tabular}

*For $\chi^{2}$ tests. 


\section{What is already known on this topic}

Eating disorders in young diabetic women are associated with earlier onset of diabetic complications

Underdosing of insulin is a common method of weight loss among diabetic women

Small studies have found no difference in prevalence of eating disorders among adolescents with and without diabetes

\section{What this study adds}

The prevalence of eating disorders was about twice as high among diabetic females aged 12-19 as that among age matched controls

Diabetic subjects with eating disorders had higher haemoglobin $\mathrm{A}_{k}$ concentrations than those without eating disorders

in the control group are high, the possibility of selection bias cannot be excluded. In addition, our diabetes sample is not truly population based, and a referral bias to the paediatric diabetes clinics may exist. Finally, a higher proportion of our control subjects were in the upper socioeconomic group than the diabetic subjects. However, we found no statistical relation between socioeconomic status and eating disorder.

Despite these limitations, our study overcomes the methodological difficulties of previous studies. The findings are both statistically and clinically meaningful, and the magnitude of the difference in prevalence is comparable with that found in previous studies with small sample sizes. ${ }^{9-13}$ The increased prevalence of eating disorders in adolescent females with type 1 diabetes may reflect an interaction between individual and environmental factors in the pathogenesis of eating disorders, similar to that observed in other high risk groups, such as competitive athletes, ${ }^{28}$ models, ${ }^{29}$ and ballet dancers. ${ }^{30}$ However, eating disorders in adolescent females with type 1 diabetes pose a particular health risk in that they are associated with impaired metabolic control and about a threefold increase in the risk of diabetic retinopathy. ${ }^{4}$ Preliminary research suggests that clinic based interventions may help to diminish disordered eating attitudes in these young women. $^{31}$ Further study is needed to determine whether intensive diabetes treatment regimens contribute to the increased risk of eating disorders in this population.

We acknowledge the time and effort of the young women who participated in our study. We also thank David Streiner and Gerald Devins for their statistical and methodological contributions and Susan Bennett and Anne Rydall for help with data collection. Finally, we thank the staff at the participating hospitals and schools, and in particular John Vadermeulen at Hamilton Health Services.

Contributors: The research was developed within the diabetes and eating disorders research group of the University Health Network, the Hospital for Sick Children, Toronto, and the University of Toronto, of which GR, DD, and MPO are senior members. The research formed part of JMJ's $\mathrm{PhD}$ thesis at the Institute of Medical Science, University of Toronto, which was supervised by GR. JMJ conducted the data collection and statistical analysis and drafted the paper. MPO and MLL provided statistical guidance. All authors contributed to the revision and editing of the paper. GR and JMJ will act as guarantors for the paper.
Funding: Medical Research Council of Canada (MA-12855), Children's Hospital of Eastern Ontario Research Institute (96/15S(E)), Genesis Foundation, and Toronto Hospital Psychiatry Research Fund.

Competing interests: None declared.

1 Striegel-Moore RH. Etiology of binge eating: a developmental perspective. In: Fairburn C, Wilson GT, eds. Binge eating. New York: Guilford, 1993:144-72

2 Fairburn CG, Beglin SJ. Studies of the epidemiology of bulimia nervosa. Am J Psychiatry 1990;147:401-8.

3 Polivy J, Herman CP, Pliner P. Perception and evaluation of body image: The meaning of body shape and size. In: Olson JP, Zanna MP, eds. Self-inference process: the Ontario symposium. Hillsdale, NJ: Erlbaum, 1990:87-114.

4 Rydall AC, Rodin GM, Olmsted MP, Devenvi RG, Daneman D. Disordered eating behavior and microvascular complications in young women with insulin-dependent diabetes mellitus. $N$ Engl J Med 1997:336:1849-54.

5 Steel JM, Young RJ, Lloyd GG, Clarke BF. Clinically apparent eating disorders in young diabetic women: associations with painful neuropathy and other complications. BMJ 1987;294:859-62.

6 Colas C, Mathieu P, Tehobroutsky G. Eating disorders and retinal lesions in type I (insulin-dependent) diabetic women. Diabetologia,1991;34:288.

7 Diabetes Control and Complications Trial (DCCT) Research Group Weight gain associated with intensive therapy in the diabetes control and complications trial. Diabetes Care 1988;11:567-73.

8 Rodin GM, Daneman D. Eating disorders and IDDM: a problemati association. Diabetes Care 1992;15:1402-12

9 Fairburn CG, Peveler RC, Davies B, Mann JI, Mayou RA. Eating disorder in young adults with insulin dependent diabetes mellitus: a controlled study. BMJ 1991:303:17-20.

10 Peveler RC, Fairburn CG, Boller I, Dunger DB. Eating disorders in adolescents with IDDM: a controlled study. Diabetes Care 1992;15: 1356-60.

11 Striegel-Moore RH, Nicholson TJ, Tamborlane WV. Prevalence of eating disorder symptoms in preadolescent and adolescent girls with IDDM. Diabetes Care 1992;15:1361-8.

12 Vila G, Nollet-Clemencon C, Vera L, Crosnier H, Robert JJ, Mouren-Simeoni MC. Etude des troubles des conduites alimentaires dans une population d'adolescentes souffrant de diabete insulinodependent. Can J Psychiatry 1993;38:606-10.

3 Mannucci E, Ricca V, Mezzani B, Di Bernardo M, Piani F, Vannini R, et al. Eating attitudes and behavior in IDDM patients: a case controlled study. Diabetes Care 1995; 18:1503-4.

14 Garner DM, Olmsted MP. Eating disorder inventory (EDI) manual. Odessa, Florida: Psychological Assessment Resources, 1984.

15 Garner DM, Olmsted MP, Bohr Y, Garfinkel PE. The eating attitudes test: psychometric features and clinical correlates. Psych Med 1982;12:871-8.

16 Johnson C. Initial consultation for patients with bulimia and anorexia nervosa. In: Garner DM, Garfinkel PE, eds. Handbook of psychotherapy for anorexia nervosa and bulimia. New York: Guilford, 1985:19-51.

17 Cooper Z, Fairburn CG. The eating disorder examination: a semi-structured interview for the assessment of the specific psychopathology of eating disorders. Int J Eat Disord 1987:6:1-8.

18 Gillery P, Labbe D, Dumont G, Vassault A. Glycohemoglobin assays evaluated in a large-scale quality-control survey. Clin Chem $1995 ; 41: 1644-8$

19 Hammer LD, Kraemer HC, Crisp AH. Standardized percentile curves of body-mass index for children and adolescents. Am J Dis Child 1991;145:259-63.

20 Williams RL, Schafer CA, Shisslak CM, Gronwaldt MA, Comerci GD. Eating attitudes and behaviours in adolescent women: Discrimination of normals, dieters, and suspected bulimics using the eating attitudes test and the eating disorder inventory. Int J Eat Disord 1986;5:879-94.

21 Steel JM, Young RJ, Lloyd GG, MacIntyre CC. Abnormal eating attitudes in young insulin-dependent diabetics. Br J Psychiatry 1989;155:515-21.

22 Rodin G, Craven J, Littlefield C, Murray M, Daneman D. Eating disorder and intentional insulin undertreatment in adolescent females with diabetes. Psychosomatics 1991;32:171-6.

23 American Psychiatric Association. Diagnostic and statistical manual of mental disorders. 4th ed. Washington, DC: APA, 1994

24 Cooper Z, Cooper PJ, Fairburn CG. The validity of the eating disorder examination and its subscales. Br J Psychiatry 1989;154:807-12.

25 Wilson GT, Smith D. Assessment of bulimia nervosa: an evaluation of the eating disorder examination. Int J Eat Disord 1989;8:173-9.

26 Rosen JC, Vara L, Wendt S, Leitenberg H. Validity studies of the eating disorder examination. Int J Eat Disord 1990;9:519-28

27 Diabetes Control and Complications Trial (DCCT) Research Group. The effect of intensive treatment of diabetes on the development and progression of long-term complications in insulin-dependent diabetes mellitus. N Engl J Med 1993;329:977-86.

28 Davis C. Body image, dieting behaviours, and personality factors: a study of high performance female athletes. Int J Sports Psychology 1992;23: 179-92.

29 Garner DM, Garfinkel PE. Sociocultural factors in the development of anorexia nervosa. Psych Med 1980;10:647-56.

30 Abraham S. Characteristics of eating disorders among young ballet dancers. Psychopathology 1996;29:223-9.

31 Olmsted MP, Rodin GM, Rydall AC, Lawson ML, Daneman D. Effect of psychoeducation on disordered eating attitudes and behaviors in young women with IDDM. Diabetes 1997;46 (suppl 1):88A.

(Accepted 24 February 2000) 